\title{
ITERATED FINE LIMITS
}

\author{
KOHUR GOWRISANKARAN
}

(Communicated by Irwin Kra)

\begin{abstract}
Let $v$ and $u$ be, respectively $n$-superharmonic and $n$-harmonic functions on the product of $n$ harmonic spaces. We prove that the iterated fine limits of $\frac{v}{u}$ exist and are independent of the order, for $\lambda$ almost every minimal boundary element where $\lambda$ represents the function $u$. As an application we prove an important property concerning the reduced function of a positive $n$ harmonic function.
\end{abstract}

\section{INTRODUCTION}

Let $\Omega_{j}, \quad j=1$ to $n$ be Brelot harmonic spaces, each space with a countable base for open sets and on each space the harmonic functions satisfy the basic axioms 1, 2, and 3 and further it is assumed that there is a potential $>0$ on each space [2]. We consider in this paper the product $\Omega_{1} \times \cdots \times \Omega_{n}$ and the $n$-hyperharmonic functions on the product, i.e. the lower semicontinuous functions that are hyperharmonic in each variable. It is known that for each positive $n$-harmonic function $u$ there corresponds a unique finite Borel measure $\lambda$ on the product of the minimal boundaries such that $u=$ $\int\left(h_{1} \ldots h_{n}\right) \lambda\left(d h_{1} \ldots d h_{n}\right)$ [4, Theorem 7]. We proved in [5, $\S 3$ and $\left.\S 4\right]$ that for a $n$-superharmonic function $v>0$ i.e. a $n$-hyperharmonic function which is not $\equiv+\infty$, and positive $n$-harmonic function of the form $u_{1}\left(x_{1}\right) u_{2}\left(x_{2}\right) \cdots$ $u_{n}\left(x_{n}\right)$, i.e. product of $n$ functions each of them harmonic $>0$ on the respective spaces $\Omega_{j}$, the iterated fine limits (in the sense specified below) exist for $\mu_{1} \times \cdots \times \mu_{n}$ almost every element of the minimal boundary where $\mu_{j}$ is the canonical measure of $\mu_{j}$ for each $j$. Here we prove the general case as indicated in the abstract. As an application we characterize the greatest $n$-harmonic minorant of (multi) reduced functions; this is a result of considerable importance in the study of the boundary behaviour of $n$-superharmonic functions.

We shall present here the proofs in the case $n=2$, the situation where $n$ is a bigger integer does not need any significant change in the proofs. We also remark that (i) these results are new even for the case of (Laplace) harmonic

Received by the editors August 11, 1988 and, in revised form, March 14, 1989.

1980 Mathematics Subject Classification (1985 Revision). Primary 31D05; Secondary 31 B25.

Key words and phrases. $n$-superharmonic, fine limits, multi-reduced function. 
functions and $\Omega_{j}$ are assumed to be discs in the plane and (ii) clearly all our proofs are valid for harmonic spaces that are more general.

Before we proceed with the proofs let us establish some notations and results that we need below. We shall assume that ${ }_{1} \Delta_{1}\left(\right.$ resp $\left.{ }_{2} \Delta_{1}\right)$ is the set of minimal positive harmonic functions on $\Omega_{1}$ (resp. $\Omega_{2}$ ) taking the value 1 at a fixed point $x_{0} \in \Omega_{1}$ (resp. $y_{0} \in \Omega_{2}$ ). To each positive 2-harmonic function $u$ there corresponds a unique finite Borel measure $\lambda$ on ${ }_{1} \Delta_{1} \times{ }_{2} \Delta_{1}$ we refer to as the canonical measure of $u$, such that $\forall(x, y) \in \Omega_{1} \times \Omega_{2}$,

$$
u(x, y)=\int h(x) h^{\prime}(y) \lambda\left(d h d h^{\prime}\right) .
$$

The spaces of positive harmonic functions on $\Omega_{j}$ and the space of positive 2-harmonic functions on the product taking the value 1 at a fixed point of the respective space are compact and metrizable in the compact open topology and the minimal elements form a $G_{\delta}$-subset and hence a polish space [2], [4]. Further every Borel subset of these sets of minimal elements is a Lusin space [6].

\section{A RESULT CONCERNING $\lambda$}

Consider the positive 2-harmonic function $u$ and the corresponding canonical measure $\lambda$. Let, for every $y \in \Omega_{2}, \quad \nu_{y}$ denote the canonical measure on ${ }_{1} \Delta_{1}$ which represents the harmonic function $x \mapsto u(x, y)$ and for brevity let us denote by $\nu_{0}$ the measure obtained when $y=y_{0}$. Since $y \mapsto \nu_{y}$ depends harmonically, the Borel subsets $\subset_{1} \Delta_{1}$ of $\nu_{y}$ measure zero are independent of $y$ in $\Omega_{2}$ [4, Lemma 7]. We also recall that we can find a Borel subset $A \subset{ }_{1} \Delta_{1}$ with $\nu_{0}\left({ }_{1} \Delta_{1} \backslash A\right)=0$ such that for all $h \in A$, the fine limit of $u(x, y) / u\left(x, y_{0}\right)$ exists as $x$ tends to $h$; this limit is locally uniform in $y \in \Omega_{2}$ and the limit function is $>0$ and harmonic on $\Omega_{2}$ [5, Lemma 2]. Let us denote the limit function by $u_{h}(\cdot) \forall h \in A$ and let $\sigma_{h}$ be the corresponding canonical measure on ${ }_{2} \Delta_{1}$. Let us recall that $\forall y \in \Omega_{2}$, the function $u_{h}(y)$ is a Radon-Nikodym derivative of the absolutely continuous part of the measure $\nu_{y}$ relative to $\nu_{0}$ [3].

However, $\nu_{y}$ and $\nu_{0}$ are mutually absolutely continuous relative to each other and we conclude that $d \nu_{y}=u_{h}(y) d \nu_{0}$. We also know that $(h, y) \mapsto$ $u_{h}(y)$ is a Borel function on ${ }_{1} \Delta_{1} \times \Omega_{2}$. This in turn implies that $h \mapsto u_{h}(\cdot)$ is a Borel function from ${ }_{1} \Delta_{1}$ with values in the space of positive harmonic functions on $\Omega_{2}$. However, the latter space is polish and we conclude that $h \mapsto u_{h}(\cdot)$ is a Lusin measurable function for every finite Borel measure on $\Delta_{1}$. In particular, we can find compact sets $K_{n}$ of ${ }_{1} \Delta_{1}$ the $\nu_{0}$-measures of which approximate that of ${ }_{1} \Delta_{1}$ such that the mapping $h \mapsto u_{h}(\cdot)$ restricted to each $K_{n}$ is continuous. Hence, we can define a unique measure $\tilde{\lambda}$ on ${ }_{1} \Delta_{1} \times{ }_{2} \Delta_{1}$ given by $d \tilde{\lambda}=\int \sigma_{h}\left(d h^{\prime}\right) \nu_{0}(d h)$, i.e. for all bounded Borel functions on ${ }_{1} \Delta_{1} \times{ }_{2} \Delta_{1}$,

$$
\int f d \tilde{\lambda}=\int_{\Delta_{1}} \int_{2 \Delta_{l}} f\left(h, h^{\prime}\right) \sigma_{h}\left(d h^{\prime}\right) \nu_{0}(d h)
$$


[1]. In particular for all $x \in \Omega_{1}, \quad y \in \Omega_{2}$ we have

$$
\begin{aligned}
\int h(x) h^{\prime}(y) d \tilde{\lambda} & =\int_{1 \Delta_{1}} \int_{2 \Delta_{1}} h(x) h^{\prime}(y) \sigma_{h}\left(d h^{\prime}\right) \nu_{0}(d h) \\
& =\int_{1 \Delta_{1}} h(x) u_{h}(y) \nu_{0}(d h) \\
& =\int_{1 \Delta_{1}} h(x) \nu_{y}(d h) \\
& =u(x, y) .
\end{aligned}
$$

But $\tilde{\lambda}$ is a finite Borel measure on ${ }_{1} \Delta_{1} \times{ }_{2} \Delta_{1}$ and by the uniqueness of the representing measure we deduce the following:

Theorem 1. The measure $\tilde{\lambda}$ constructed above is indeed the canonical measure which represents the 2-harmonic function $u$; i.e. $\tilde{\lambda}=\lambda$.

\section{3. "ITERATED FINE LIMITS" of $v(x, y) / u(x, y)$}

Let us now consider a 2-superharmonic function $v>0$ on the product space. As introduced in the above section we consider $u_{h}$, for all $h \in A$ for the fixed positive 2-harmonic function $u$. Let $\beta_{2}$ be a countable base for open sets of $\Omega_{2}$ consisting of regular domains. Using the results in [5] we conclude that there exists a Borel set $A_{v} \subset A$ with $\nu_{0}\left(A-A_{v}\right)=0$ (this implies also $\left.\nu_{0}\left({ }_{1} \Delta_{1} \backslash A_{v}\right)=0\right)$ such for all $h \in A_{v}$ the following is valid. The function

$$
y \mapsto \text { fine } \limsup _{x \rightarrow h} \frac{v(x, y)}{u(x, y)}=\left[\text { fine } \lim \sup \frac{v(x, y)}{u\left(x, y_{0}\right)}\right] \frac{1}{u_{h}(y)}
$$

is a $\left(\beta_{2}\right)$ nearly superharmonic function on $\Omega_{2}$; and the above function is Borel in the variables $h$ and $y$ together. Further, if $v$ is a positive 2-harmonic function the above limit function in this case is defined for all $y \in \Omega_{2}$ and $v_{h}(y)$ is harmonic for all $h \in A_{v}$. We now have

Theorem 2. Let $w>0$ be a 2-harmonic function on the product. Then the iterated fine limits of $w / u$ exist for $\lambda$ almost every $\left(h, h^{\prime}\right)$ in the minimal boundary and is independent of the order of iterated limits. Further, the (iterated) of the canonical measure of $w$ relative to $\lambda$. In particular, if $w / u$ is bounded (or quasi-bounded) then the canonical measure of $w$ is precisely $\phi_{w} d \lambda$.

Proof. As in the remark preceding the theorem we choose a Borel set $A_{w} \subset$ $A \subset{ }_{1} \Delta_{1}$ such that $\nu_{0}\left(A_{w}\right)=\nu_{0}\left({ }_{1} \Delta_{1}\right)$ and consider the function $w_{h} / u_{h}$. As in [5, Th. 2], we conclude that $C_{w} \subset A_{w} \times{ }_{2} \Delta_{1}$ defined by

$$
C_{u \prime}=\left\{\left(h, h^{\prime}\right): \text { fine } \lim _{y \rightarrow h^{\prime}} w_{h}(y) / u_{h}(y) \text { exists in } \mathbf{R}\right\}
$$

is a Borel subset of $A_{w} \times{ }_{2} \Delta_{1}$ and that the limit function is Borel on $C_{w}$. However, for every $h \in A_{w}$, the section of $C_{w}$ through $h$ is of full $\sigma_{h}$ measure (recall that $\sigma_{h}$ is the canonical measure on ${ }_{2} \Delta_{1}$ representing $\left.u_{h}(\cdot)\right)$. It follows 
that $\tilde{\lambda}\left({ }_{1} \Delta_{1} \times{ }_{2} \Delta_{1}-C_{w}\right)=0$. We conclude from Theorem 1 that $\lambda\left(C_{w}\right)=$ $\lambda\left({ }_{1} \Delta_{1} \times{ }_{2} \Delta_{1}\right)$ and thereby the existence of the iterated limit $\lambda$ almost everywhere. The rest of the proof is completed exactly as in [5, Th. 6].

Once again following the steps similar to the proof of Theorem 2 and the techniques in [5] we prove the

Theorem 3. Let $v>0$ be a 2-superharmonic function and $u>0$ and 2harmonic function. Then for $\lambda$ almost every $\left(h, h^{\prime}\right)$ in the minimal boundary the following

$$
\phi_{v}\left(h, h^{\prime}\right)=\text { fine } \limsup _{y \rightarrow h^{\prime}}\left\{\left[\text { fine } \limsup _{x \rightarrow h} \frac{v(x, y)}{u(x, y)}\right]^{\wedge}\right\}
$$

exists where $\psi^{\wedge}$ is the lower semi-continuous regularization of $\psi$. Further, $\phi_{v}\left(h, h^{\prime}\right) d \lambda$ is the canonical measure of the quasi u-bounded component of the greatest 2-harmonic minorant of $v$. In particular, this function $\phi_{v}$ equals $\lambda$ almost everywhere the function obtained by reversing the role of the variables.

Remark 1. Let us define

$$
\psi_{v}\left(h, h^{\prime}\right)=\text { fine } \liminf _{y \rightarrow h^{\prime}}\left\{\left[\text { fine } \liminf _{x \rightarrow h} \frac{v(x, y)}{u(x, y)}\right]^{\wedge}\right\}
$$

It is very simple to verify directly that this function equals $\phi_{v}\left(h, h^{\prime}\right)$ of the Theorem on a set of full $\lambda$ measure.

Remark 2. By abuse of language we will refer to the function $\phi_{v}$ associated with $v$ as "the iterated fine limit" of $v$. We note this function is defined only up to a Borel set of $\lambda$ measure 0 . We also note that the above "iterated fine limit" process is positively homogeneous and additive.

\section{AN APPLICATION}

Let $E \subset \Omega_{1} \times \Omega_{2}$ and $f \geq 0$ on $\Omega_{1} \times \Omega_{2}$. We denote the (multi) reduced function

$$
M R(E, f)=\operatorname{Inf} .\left\{\begin{array}{ll}
v \geq 0 & \text { 2-hyperharmonic } \\
v \geq f & \text { on } E
\end{array}\right\} .
$$

If $E$ is open and $f=v$ a 2-superharmonic function $>0$ then $M R(E, v)$ is 2-superharmonic and equals $v$ on $E$. We have the following result concerning the greatest 2-harmonic minorant of such functions.

Theorem 4. Let $\omega \subset \Omega_{1} \times \Omega_{2}$ be a nonvoid open subset and $u>0$ a 2-harmonic function with the canonical measure $\lambda$. Let $w$ be the greatest 2-harmonic minorant of $M R(\omega, u)$ and $\nu$ its canonical measure. Then $\exists$ a Borel set $B \subset{ }_{1} \Delta_{1} \times{ }_{2} \Delta_{1}$ such that

$$
w(x, y)=\int_{B} h(x) h^{\prime}(y) \lambda\left(d h d h^{\prime}\right),
$$

i.e. $d \nu=\chi_{B} d \lambda$. 
Proof. Clearly $w \leq u$ which gives us the inequality $\nu \leq \lambda$ and hence $d \nu=$ $g d \lambda$ for some Borel function $g, \quad 0 \leq g \leq 1$. By Theorem 2 we know that $g\left(h, h^{\prime}\right)=$ iterated fine limit of $w / u$ for $\lambda$ almost every $\left(h, h^{\prime}\right)$. We note further that $g$ is also the "iterated fine limit" of $M R(\omega, u) / u, \lambda$ almost everywhere. Let for every $\alpha, \quad 0 \leq \alpha<1, \quad D_{\alpha}=\left\{\left(h, h^{\prime}\right): g\left(h, h^{\prime}\right) \leq \alpha\right\}$ and $D=\cup D_{\alpha}=\left\{\left(h, h^{\prime}\right): g\left(h, h^{\prime}\right)<1\right\}$. If $D$ is of $\lambda$-measure 0 there is nothing to prove and in this case $w=u=M R(\omega, u)$. Let us hence consider $\alpha<1$ such that $\lambda\left(D_{\alpha}\right)>0$. Let $u_{\alpha}$ be the harmonic function $=\int_{D_{\alpha}} h h^{\prime} \lambda\left(d h d h^{\prime}\right)$; i.e. with canonical measure the restriction of $\lambda$ to $D_{\alpha}$. Consider the 2-superharmonic function $M R\left(\omega, u_{\alpha}\right)=q+p$ where $q$ is the greatest 2-harmonic minorant of $M R\left(\omega, u_{(x)}\right)$. Consider

$$
\begin{aligned}
\phi\left(h, h^{\prime}\right) & =\frac{\text { "iterated fine limit" }}{x \rightarrow h, y \rightarrow h^{\prime}} \quad M R\left(\omega, u_{\alpha}\right) / u_{\alpha} \\
& =\frac{\text { iterated fine limit }}{x \rightarrow h, y \rightarrow h^{\prime}} \quad q(x, y) / u_{\alpha}(x, y) .
\end{aligned}
$$

Expressing $M R\left(\omega, u_{\alpha}\right) / u=\left[M R\left(\omega, u_{\alpha}\right) / u\right]\left[u / u_{\alpha}\right]$, and recalling that the iterated fine limit of $u_{\alpha} / u=1$ for $\lambda$ a.e. element of $D_{\alpha}$, we get the inequality

$$
\phi\left(h, h^{\prime}\right)=\left(\text { iterated fine } \lim M R\left(\omega, u_{\alpha}\right) / u\right)(1) \text { for } \lambda \text { a.e. }\left(h, h^{\prime}\right) \in D_{\alpha}
$$

so

$$
\phi\left(h, h^{\prime}\right)=g\left(h, h^{\prime}\right) \leq \alpha \text { for } \lambda \text { almost every }\left(h, h^{\prime}\right) \in D_{\alpha} .
$$

Now $q \leq u_{\alpha}$ and hence the canonical measure of $q$ is concentrated on $D_{\alpha}$, $D_{\alpha}$ being the carrier of the canonical measure of $u_{\alpha}$. Also

$$
q=\int_{B_{\alpha}} h h^{\prime} \phi\left(h, h^{\prime}\right) d \lambda .
$$

We conclude first that $q \leq \alpha u_{\alpha}$. Now, we have,

$$
\begin{aligned}
q+p=M R\left(\omega, u_{\alpha}\right) & =M R\left[\omega, M R\left(\omega, u_{\alpha}\right)\right] \\
& \leq M R(\omega, q)+M R(\omega, p) \\
& \leq M R\left(\omega, \alpha u_{\alpha}\right)+p \\
& =\alpha(q+p)+p=\alpha q+p(1+\alpha) .
\end{aligned}
$$

It follows that $q \leq$ the greatest 2-harmonic minorant of the right side which is $\alpha q$. Since $\alpha<1$, we conclude that $q \equiv 0$. Hence, we have,

$$
\text { "iterated fine limit" } M R\left(\omega, u_{\alpha}\right) / u_{\alpha}=0 \quad \lambda \text { a.e. on } D_{\alpha} .
$$

Now,

$$
M R(\omega, u) \leq M R\left(\omega, u_{\alpha}\right)+\left(u-u_{\alpha}\right)
$$

and

$$
\begin{gathered}
0 \leq \text { "iterated fine limit" } M R(\omega, u) \leq \text { "iterated finelim" } M R(\omega, u) / u_{\alpha} \\
\leq 0+0=0 \quad \lambda \text { a.e. on } D_{\alpha} .
\end{gathered}
$$


This implies that $g=0 \lambda$ almost everywhere on $B_{\alpha}$. Since $D$ is the increasing union of $D_{\alpha_{n}}$ where $\alpha_{n} \uparrow 1$ we conclude that $g=0 \lambda$ almost everywhere on $D$. The required set $B={ }_{1} \Delta_{1} \times{ }_{2} \Delta_{1}-D$. The proof is complete.

Corollary. A similar result for $M R(E, u)$ is valid for any arbitrary $E \subset \Omega_{1} \times \Omega_{2}$.

This is deduced easily by using the fact that $M R(E, u)$ is the pointwise decreasing limit of the 2-superharmonic functions $M R(\omega, u)$ for all open sets $\omega \supset E$.

\section{BIBLIOGRAPHY}

1. N. Bourbaki, Intégration, chapitre 5, "Intégration des Mesures".

2. M. Brelot, Seminaire de Theorie du Potential, I. H. P. Paris, 1958.

3. K. Gowrisankaran, Fatou-Naim-Doob limit theorems in the axiomatic system of Brelot, Ann. Inst. Fourier (Grenoble) 16 (1966), 455-467.

4. __ Multiply Harmonic Functions, Nagoya Math. J. 28 (1966), 27-48.

5. __ Iterated fine limits and iterated non-tangential limits, Trans. Amer. Math. Soc. 173 (1972), 71-92.

6. L. Schwartz, Radon measures on general topological spaces, TIFR Monographs Oxford Univ. Press, 1973.

McGill University, Department of Mathematics and Statistics, 805 Sherbrooke West, Montreal, H3A 2K6, Canada 